\title{
Structural Stress Analysis of $32 \times 32$ InSb Infrared Focal Plane Array with Underfill
}

\author{
Qingduan Meng ${ }^{1, *}$, Junxian $\mathrm{Li}^{1}$, Yanqiu $\mathrm{Lv}^{2}$ and Weiguo $\mathrm{Sun}^{2}$ \\ ${ }^{1}$ School of Electronic Information Engineering, Henan University of Science and Technology, Luoyang 471003, China \\ ${ }^{2}$ China Airborne Missile Academy, Luoyang 471009, China
}

\begin{abstract}
Based on viscoplastic Anand's model, the structural stress of $8 \times 8 \mathrm{InSb}$ infrared focal plane array detector with underfill is systematically analyzed by finite element method, and the impacts of design parameters including indium bump diameters, heights on both Von Mises stress and its distribution are discussed in this manuscript. Simulation results show that for the given indium bump height, the maximum stress existing in InSb chip is almost unchanged with reduced indium bump diameters, and with thicker indium bump, the stress is smaller, when the indium bump height is over than $15 \mu \mathrm{m}$, the stress reduces slowly with decreased standing off height. When the InSb IRFPA format increases from $8 \times 8$ to $32 \times 32$, the maximal Von Mises stress increases from $500 \mathrm{MPa}$ to $900 \mathrm{Mpa}$, almost twice over, and is not proportional to array format scale.
\end{abstract}

Keywords: Finite element method, focal plane array, InSb, structural stress.

\section{INTRODUCTION}

Indium antimonide ( $\mathrm{InSb}$ ) pn junctions are widely used as infrared photovoltaic detectors and imaging sensors for the $3-5 \mu \mathrm{m}$ spectral range $[1,2]$. Photovoltaic diodes are usually placed in rectangular array or square array, then via indium bumps, assembled to a silicon readout integrated circuits (ROIC), after that, underfill is dispensed between InSb die and Si ROIC substrate for increasing the reliability of flip-chip packaging. To achieve the lowest level of electronic noise to approach the photon noise limit, it is necessary to cryogenically cool the infrared focal plane array (IRFPA) to liquid nitrogen temperature $(77 \mathrm{~K})$. Therefore, fracture in InSb chip usually occurs with the rapid temperature dropping process, due to the mismatch in the coefficients of thermal expansion between the silicon multiplexer and the InSb detector array, which will cause mechanical stress/strain on both indium bumps and $\mathrm{InSb}$ chip with thickness less than one diffusion length about $8 \mu \mathrm{m}$. In order to reduce the InSb chip fracture possibility and improve the yield, it is necessary to analyze the induced thermal strain and stress values and its distribution in $\mathrm{InSb}$ chip. At present time, the thermo-mechanical reliability of flip-chip assemblies is usually assessed by finite element simulations in conjunction with experimental verification, that is, based on the strain and stress analysis, the structure reliability is assessed $[3,4]$.

In 2009, Rui Wu Chang research group delivers the constitutive relations of indium in extreme-temperature in Anand's model [5], a rate and temperature dependent consti-

\footnotetext{
*Address correspondence to this author at the School of Electronic Information Engineering, Henan University of Science and Technology, Luoyang 471003, China; Tel: +86-379-64231157; Office: +86-13525454409; Fax: +86-379-64231910;

E-mail: qdmengly@mail.haust.edu.cn,qdmengly@163.com
}

tutive model, applied to represent the inelastic deformation behavior of indium at low temperatures down to $-150^{\circ} \mathrm{C}$. Based on the above research results, the structural stress of $8 \times 8 \mathrm{InSb}$ array detector with underfill dependent on both indium bump diameters and thickness is systematically researched by finite element method, for finding the optimal structural sizes to avoid thermal stress concentration in $\mathrm{InSb}$ chip.

\section{MODEL CREATION AND PARAMETERS SELECTION}

When creating the finite element analysis model of $\mathrm{InSb}$ IRFPA detector, indium bumps are assumed to be octagonal prisms, and no defects exist in the whole device. InSb IRPFA is composed of InSb photodiode arrays, indium bumps array, Si ROIC and underfill encapsulant materials, just as shown in Fig. (1). InSb chip dimensions are $1600 \mu \mathrm{m} \times 1600 \mu \mathrm{m} \times 8 \mu \mathrm{m}$, and attached to Si ROIC substrate with $32 \times 32$ indium bumps array, Si ROIC substrate dimensions are $1700 \mu \mathrm{m} \times 1700 \mu \mathrm{m} \times 300 \mu \mathrm{m}$, indium bumps height has been fixed at $24 \mu \mathrm{m}$ with $50 \mu \mathrm{m}$ pitch, and its diameter increases from $20 \mu \mathrm{m}$ to $36 \mu \mathrm{m}$ in step of $4 \mu \mathrm{m}$. In order to describe the mesa structure locating between neighboring detectors elements, a triangular prism, whose cross section area sides length are $5.66 \mu \mathrm{m}, 5.66 \mu \mathrm{m}$ and $8 \mu \mathrm{m}$, respectively, are employed. Using the geometrical symmetry, only one eighth of the overall package is modeled.

InSb chip and Si ROIC substrate are both considered to be linear elastic materials. All the employed parameters are listed in Table 1, where $\mathrm{E}$ is the Young's modulus, $\mu$ is the Poisson's ratio, and CTE is the coefficient of thermal expansion (CTE). Flip chip process is completed at $370 \mathrm{~K}$, after that, the selected underfill is dispensed between InSb chip and Si ROIC substrate, the glass transition temperature of underfill is $408 \mathrm{~K}$, and the curing process is also operated at 


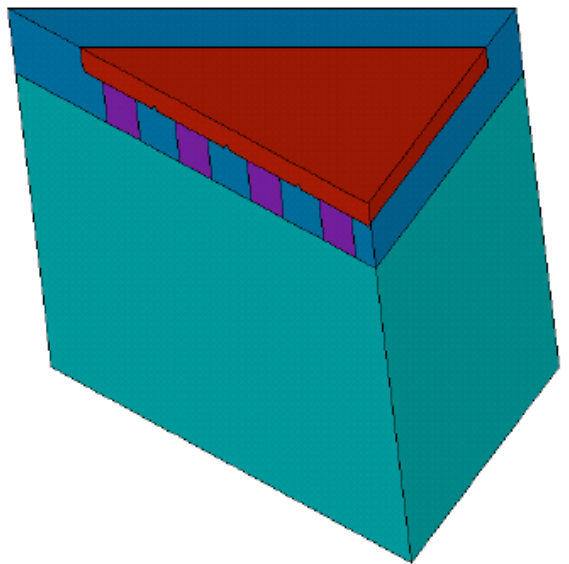

Fig. (1). Three dimensional model of infrared focal plane array device.

Table 1. Linear Elastic Material Parameters

\begin{tabular}{|c|c|c|c|}
\hline Materials & $\begin{array}{c}\text { Elastic Modulus } \\
{[\mathbf{E} / \mathbf{M P a}]}\end{array}$ & $\begin{array}{c}\text { Poison's } \\
\text { Ratio }[\boldsymbol{\mu}]\end{array}$ & $\begin{array}{c}\text { CTE } \\
{\left[\boldsymbol{\alpha}_{I} \mathbf{p p m} / \mathbf{K}\right]}\end{array}$ \\
\hline \hline Si ROIC & 163000 & 0.28 & 2.50 \\
\hline InSb chip & 409000 & 0.35 & 5.04 \\
\hline Indium bump & 10600 & 0.45 & 33.00 \\
\hline Underfill & 9000 & 0.3 & 26.00 \\
\hline
\end{tabular}

$408 \mathrm{~K}$, at this temperature, no residual stress is assumed to exist within the package. In the simulation, the temperature is gradually reduced from $408 \mathrm{~K}$ to $77 \mathrm{~K}$, which causes thermal stresses to develop. During the temperature drop, no transient heat transfer is considered and the temperature within the model is assumed to be uniform. After about 71 seconds, increments are taken to lower the temperature to ensure the accuracy. Horizontal displacements normal to the symmetrical surfaces are set to zero, at the same time, the displacement of Si ROIC substrate bottom surface center point remains zero.

Indium bump deformation is strongly temperature and time dependent, and associated with the irreversible, temperature and rate dependent inelastic characteristics, which are known to be viscoplastic [6-8]. Here Anand's viscoplastic constitutive model has been used to describe mechanics of constitutive relationship of indium bump [9, 10].

Underfill is an epoxy-based polymer exhibiting time-andtemperature dependent viscoelastic properties. The viscoelastic behavior of underfill can be analyzed using the timetemperature superposition principle, which is based on the empirical observation that the viscoelastic behavior of polymer materials at a reference temperature $T_{0}$ can be related to the behavior at another temperature $T$ by changing the experimental time or frequency scale [11-13]. Generally speaking, from the polymers with the glass transition temperature $T_{0}$, the shift factor $\log \alpha_{T}$ was found to be approximately the Arrhenius equation

$$
\log \alpha_{T}=A+\frac{B}{C_{2}+T_{A B S}}
$$

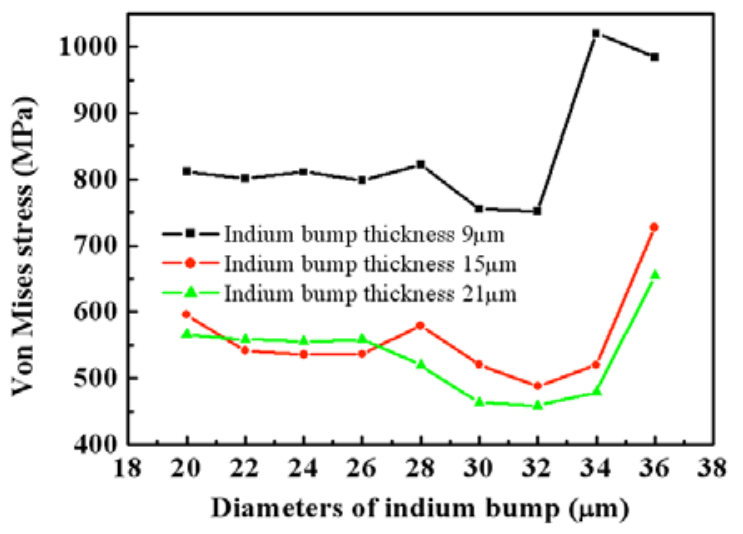

Fig. (2). Von Mises stress maximum values of $8 \times 8 \mathrm{InSb}$ as a function of vaired indium bump diameter with different indium bump heights.

where $\mathrm{A}$ and $\mathrm{B}$ are constants, $T_{A B S}$ is the absolute temperature in degrees Kelvin. Using the time-temperature superposition experimental results, it is possible to construct a master curve at an arbitrary reference temperature which extends the relaxation modulus beyond the range of laboratory scale in either the time or the frequency domain. Here the glass transition temperature of underfill $T_{g}$ is selected as the reference temperature, A Prony series was used to fit the master relaxation curve and this series is of the form

$G(t)=G_{\infty}+\sum_{i=1}^{n_{G}} G_{i} e^{-\frac{t}{\tau_{i}}}$

Where $G_{\infty}$ represents the equilibrium modulus after the under fill is fully relaxed; $G_{i}$ and $\tau_{i}$ are the Prony efficient and relaxation time for each element, respectively. The Prony coefficients for the master curve are given in Table 2 [14, 15].

\section{SIMULATION RESULTS ANALYSIS}

Two-step method is used to research stress and its distribution in $32 \times 32 \mathrm{InSb}$ IRFPA employing finite element method for time saving. First, a small $8 \times 8 \mathrm{InSb}$ IRFPA is systematically studied by varying indium bump diameters, standoff heights in suitable range, simulation results show that as the diameters of indium bump decrease from $32 \mu \mathrm{m}$ to $20 \mu \mathrm{m}$ in step of $2 \mu \mathrm{m}$, the maximum stress existing in $\mathrm{InSb}$ chip increases slowly from $751 \mathrm{MPa}$ to $812 \mathrm{MPa}$ with $9 \mu \mathrm{m}$ indium bump height, and for thicker indium bump, such as $15 \mu \mathrm{m}$ and $21 \mu \mathrm{m}$, the maximal Von Mises stress in the InSb chip versus indium bump diameters almost have the same vary tendency, just like the curve is shifted down some values. When the height of indium bump increases from $9 \mu \mathrm{m}$ to $21 \mu \mathrm{m}$ in step of $6 \mu \mathrm{m}$, the maximal stress in InSb chip first reduces sharply from $800 \mathrm{MPa}$ to $500 \mathrm{MPa}$, then almost retains constant, just as shown in Fig. (2). Here the temperature of InSb IRFPA is gradually reduced from $370 \mathrm{~K}$ to $77 \mathrm{~K}$ in 71 seconds, and the underfill is assumed to be linear elastic properties. Apparently, with thicker indium bump height, the maximal Von Mises stress in InSb chip is lower. To learn the effect from indium bump diameters on the maximal Von Mises stress in InSb chip, and taking manufacturing precision into consideration, here the indium bump height is selected as $24 \mu \mathrm{m}$ for smaller stress. 
Table 2. Relaxation Parameters of Viscoelectic Maxwell Model for Underfill

\begin{tabular}{|c|c|c|}
\hline $\mathbf{i}$ & $\mathbf{C}_{\mathbf{i}}$ & $\mathbf{T}_{\mathbf{i}}$ \\
\hline \hline 1 & 0.0031 & $2.1230 \times 10^{-10}$ \\
\hline 2 & 0.0299 & $4.5738 \times 10^{-9}$ \\
\hline 3 & 0.0817 & $9.8541 \times 10^{-8}$ \\
\hline 4 & 0.1158 & $2.1230 \times 10^{-7}$ \\
\hline 5 & 0.2264 & $4.5738 \times 10^{-6}$ \\
\hline 6 & 0.2413 & $9.8541 \times 10^{-5}$ \\
\hline 7 & 0.2155 & $2.1230 \times 10^{-4}$ \\
\hline 8 & 0.0676 & $4.5738 \times 10^{-2}$ \\
\hline 9 & 0.0145 & 9.8541 \\
\hline 10 & 0.0042 & $2.1230 \times 10^{2}$ \\
\hline
\end{tabular}

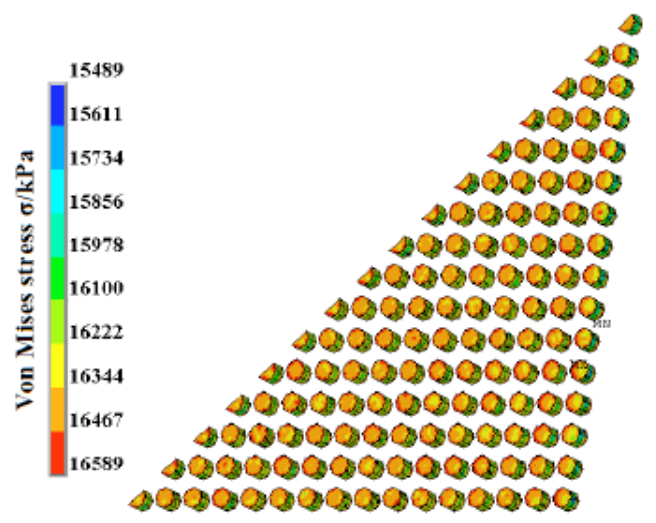

Fig. (3). Von Mises stress distribution of indium bumps array with $32 \times 32$ format.

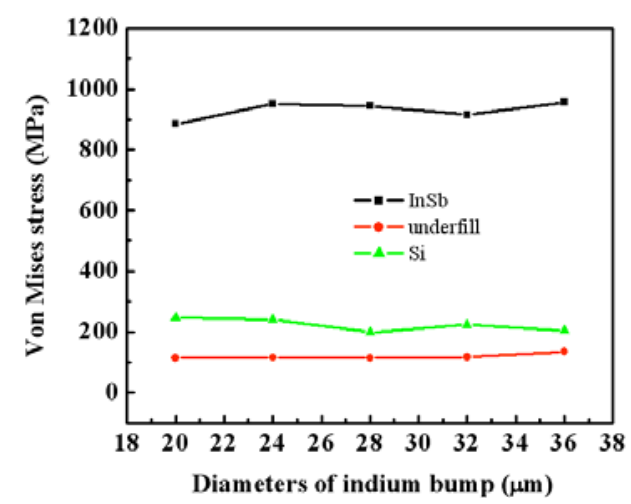

Fig. (4). Von Mises stress maximum values of $32 \times 32 \mathrm{InSb}$ chip as a function of InSb thickness with varied indium bump standoff height.

The glass transition temperature of the selected underfill is $408 \mathrm{~K}$, and the curing process is also operated at $408 \mathrm{~K}$ for some time period, at this temperature, no residual stress is assumed to exist within the package. When temperature of $32 \times 32 \mathrm{InSb}$ IRFPA is gradually reduced from $408 \mathrm{~K}$ to $77 \mathrm{~K}$ in 71 seconds, Von Mises stress distribution of indium bumps is illustrated in Fig. (3). The Von Mises stress differ-

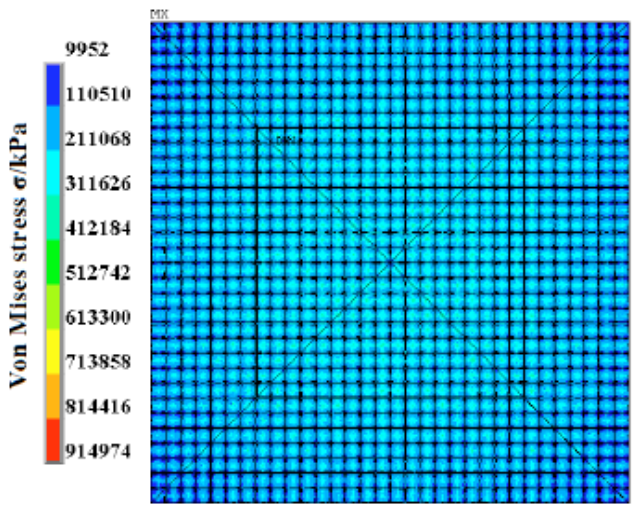

Fig. (5). Von Mises stress distribution of InSb chip bottom surface.

ence (1.1 MPa) among indium arrays is so small that the Von Mises stress distribution is assumed to be uniform. The maximal Von Mises stress locating on the indium bump top surface locating at the twelfth row and eighth column intersection. Apparently, with the underfill dispensed among the gap between InSb chip and Si ROIC substrate, the stress in indium bump array redistributes, and its distribution is well uniformed.

In order to learn the effect from the indium bump diameter to Von Mises stress and its distribution in InSb chip, here a typical structure with InSb thickness $8 \mu \mathrm{m}$, indium bump thickness $24 \mu \mathrm{m}$ is selected. While taking account of manufacturing precision the diameters of indium bump reduces from $36 \mu \mathrm{m}$ to $20 \mu \mathrm{m}$ in step of $4 \mu \mathrm{m}$, the simulation results are shown in Fig. (4). With indium bump height is fixed at $24 \mu \mathrm{m}$, the maximal Von Mises stress in the InSb chip fluctuates at $900 \mathrm{Mpa}$, it seems that the stress is not dependent on the indium bump diameter, and the stress in underfill and $\mathrm{Si}$ ROIC also shows the similar varying tendency. Compared with the $8 \times 8$ InSb IRFPA array with underfill, the underfill is described with linear elastic properties, in a $32 \times 32 \mathrm{InSb}$ IRFPA array with viscoelastic properties underfill, the stress dependent on indium bump diameter is not obvious. So in the simulation process, omitting the underfill curing effect is not accurate, especially in larger indium bump diameter range.

In the whole InSb IRFPA detector, the maximal Von Mises stress, about $900 \mathrm{MPa}$, appears on InSb chips edges, which is farthest to the neutral point, and is vertical to InSb top surface, the minimal Von Mises stress, about $16 \mathrm{MPa}$, locating in indium bump array, and the stress in underfill encapsulant is about $114 \mathrm{MPa}$, almost 7 times of that in indium bump array, this is not proportional to the support area, though their Young's modulus, Poisson's ratio, and coefficient of thermal expansion are comparable, the stress in $\mathrm{Si}$ ROIC is about $200 \mathrm{MPa}$, slightly larger than that in underfill. All these are distinct from the Von Mises stress distribution in $8 \times 8 \mathrm{InSb}$ array detector without underfill, where the Von Mises stress distribution in all the contacting areas is uniform and concentrated, and its maximal stress is also located in contacting area between InSb chip and indium bump.

When indium diameter, height and InSb chip thickness are set to $32 \mu \mathrm{m}, 24 \mu \mathrm{m}$, and $8 \mu \mathrm{m}$, Von Mises stress distribution of InSb chip, underfill and Si ROIC is shown in Figs. (57), respectively. Apparently, Von Mises stress existing on 


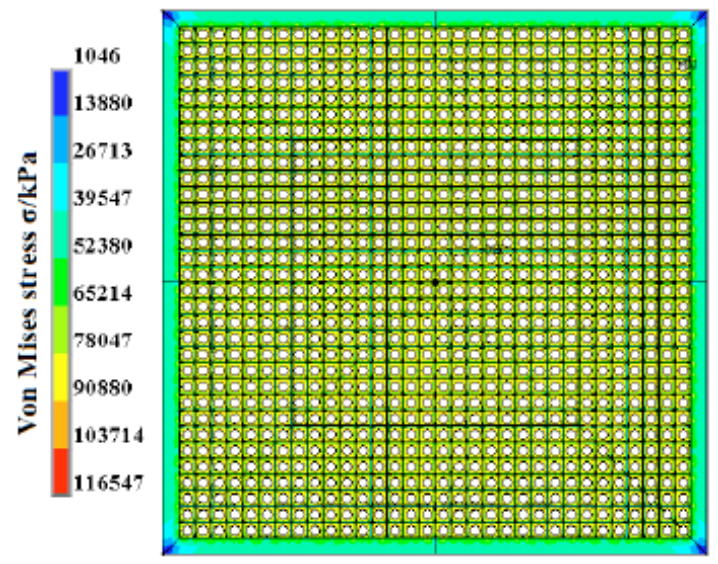

Fig. (6). Von Mises stress distribution of underfill top surface.

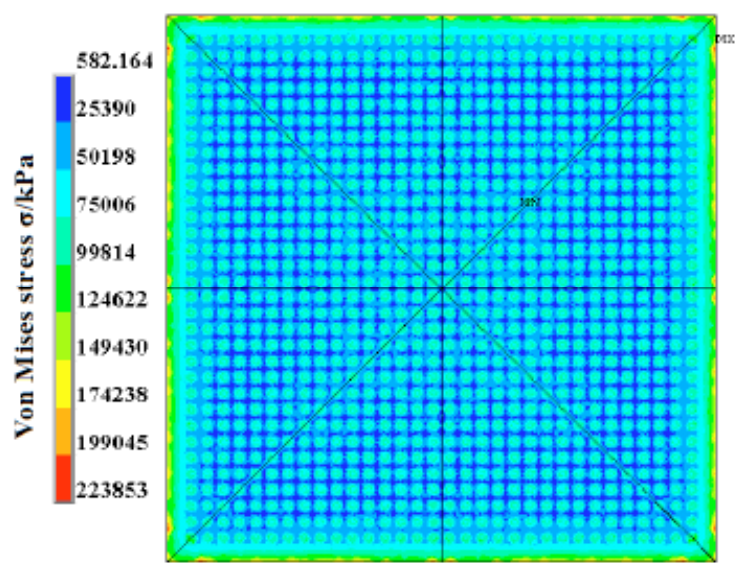

Fig. (7). Von Mises stress distribution of Si-CMOS readout integrated circuit.

contact areas between InSb chip and indium bumps is concentrated and uniform, its stress value is about $350 \mathrm{MPa}$, larger than that of its surrounding areas. The maximal stress value about $915 \mathrm{MPa}$ situates at the edge far away from neutral point, where the InSb chip is confined by its surrounding underfill encapsulate and does not shrink freely. The minimal stress value about $9 \mathrm{MPa}$ locates on the trough intersection zones. There is an obvious stress gradient from contact section center to non-contact region.

Von Mises stress distribution in underfill is shown in Fig. (6). On the whole region under InSb chip, the stress distribution is uniform, and its stress value is about $80 \mathrm{MPa}$, but around indium bump octahedral shell, the stress is apparently enlarged with maximal value $116 \mathrm{MPa}$, and its minimum appears on the intersect region of convex triangular prisms, which is made of underfill locating in the troughs etching in InSb chip for isolating the neighboring detector cells. At the four corners of underfill top surface, Von Mises stress is small and is about $1 \mathrm{MPa}$, here the underfill can shrink freely. Compared with indium bump array, apparently the underfill bears most thermal strain, for its stress is almost six times as large as stress in indium bump array.

Von Mises stress distribution in Si ROIC is almost concentrated strongly on the contacting areas between indium bumps and Si ROIC, around the contacting areas, the Von Mises is so small that it is ignorable, besides, on the quadri- lateral borders of Si ROIC top surface, there are several noncontinuous spots where Von Mises stress are the largest, especially near the corner of Si ROIC top surface, just as shown in Fig. (7). This is distinctly different from the stress distribution in the structure without underfill, where the stress distribution on the quadrilateral borders of Si ROIC top surface is ignorable [16].

\section{CONCLUSIONS}

Crack appearing in the InSb chip limited the InSb IRFPA final yield. Based on finite element analysis, impacts on Von Mises stress and its distribution in the InSb IRFPA from the structure parameters, including indium bump diameters and heights, are completely discussed in this manuscript. Simulation results show that the maximal Von Mises value existing in InSb chip is almost not dependent on indium bump diameters, and are mainly determined by standoff height, when the indium bump height is over $15 \mu \mathrm{m}$, the stress reduces slowly with decreased standing off height. When the InSb IRFPA format increases from $8 \times 8$ to $32 \times 32$, the maximal Von Mises stress increases from $500 \mathrm{MPa}$ to $900 \mathrm{MPa}$, which is almost twice over.

\section{ACKNOWLEDGEMENTS}

This research is supported by Science Foundation of Chinese Aeronautics industry.

\section{REFERENCES}

[1] Y. Gau, L. Dai, S. Yang et al., " $256 \times 256$ InSb Focal Plane Arrays," Proc. SPIE, vol. 4078, 2003, pp. 467-479.

[2] W. Parrish, J. Blackwell, G. Kincaid, and R. Paulson, "Low-Cost high performance InSb $256 \times 256$ Infrared Camera", Proc. SPIE, vol. 1540, 1991, pp. 274-284.

[3] J. Pang and D. Chong, "Flip chip on board solder joint reliability analysis using 2-D and 3-D FEA models", IEEE Trans. Adv. Pack, vol. 24, pp. 499-506, 2001.

[4] Z. Zhang and C. P. Wong, "Recent advances in flip-chip underfill: Materials, process, and reliability", IEEE Trans. Adv. Pack, vol. 27, pp. 515-523, 2004.

[5] R. Chang and F. Patrick Mccluskey, "Constitutive relations of indium in extreme temperature electronic packaging based on Anand Model", J. Electron. Mater., vol. 38, pp.1855-1859, 2009.

[6] S. Kim and H. Ledbetter, "Low-temperature elastic coefficients of polycrystalline indium ", Mater. Sci. Eng., A, vol. 252, pp.139$143,1998$.

[7] E. Hermida, D. Melo, J. Aguiar and D. Lopez, "Temperature dependence of the viscoelastic response of In, Sn and In-Sn alloys", $J$. Alloys Compd., vol. 310, pp. 91-96, 2000.

[8] R. Reed, C. McCowan and R. Walsh, "Tensile strength and ductility of indium”, Mater. Sci. Eng., vol. 102, pp. 227-236,1988.

[9] G. Wang, Z. Cheng, K. Becker and J. Wilde, "Applying anand model to represent the viscoplastic deformation behavior of solder alloys", J. Electron. Pack, vol. 123, pp.247-253, 2001.

[10] J. Wilde, K. Becker, M. Thoben, W. Blum, T. Jupitz, G. Wang and Z. Cheng, "Rate dependent constitutive relations based on Anand model for 92.5Pb5Sn2.5Ag solder", IEEE Trans. Adv. Pack, vol. 23, pp.408-414, 2000.

[11] M. Williams, R. Landel, J. Ferry, "The temperature dependence of relaxation mechanisms in amorphous polymers and other glassforming liquids", J. Am. Chem. Soc., vol. 77, pp. 3701-3707,1955.

[12] J. Ferry, Viscoelastic Properties of Polymers, 3rd ed., New York: John Wiley and Sons, 1980.

[13] I. Ward, D. Hadley, An Introduction to the Mechanical Properties of Solid Polymers, New York: John Wiley and Sons, 1997.

[14] T. Yeung, and M. Yuen, "Viscoelastic Analysis of IC Packaging Warpage", ASME S., Model. Sim.. Emerg. Electron. Pack, EEP, vol. 17, pp. 101-107,1996. 
[15] O. Narayanaswamy, "A Model of structural relaxation in glass", $J$. Am. Ceram. Soc. vol. 54, pp. 491-498, 1971.
[16] Q. Meng, X. Zhang, X. Zhang and W. Sun, "Finite element analysis on structural stress of $8 \times 8 \mathrm{InSb}$ infrared focal plane array", Appl. Mech. Mater. vol. 33-34, pp. 207-211, 2010.

Received: October 06, 2010

Revised: December 24, 2010

Accepted: May 16, 2011

(c) Meng et al.; Licensee Bentham Open.

This is an open access article licensed under the terms of the Creative Commons Attribution Non-Commercial License (http://creativecommons.org/licenses/by-nc/3.0/) which permits unrestricted, non-commercial use, distribution and reproduction in any medium, provided the work is properly cited. 